\title{
Stability Analysis for Travelling Wave Solutions of the Olver and Fifth-Order KdV Equations
}

\author{
A. R. Seadawy, ${ }^{1,2}$ W. Amer, ${ }^{1}$ and A. Sayed ${ }^{2}$ \\ ${ }^{1}$ Mathematics Department, Faculty of Science, Taibah University, Al-Ula 41921-259, Saudi Arabia \\ ${ }^{2}$ Mathematics Department, Faculty of Science, Beni-Suef University, Beni-Suef, Egypt \\ Correspondence should be addressed to A. R. Seadawy; aly742001@yahoo.com
}

Received 8 November 2013; Revised 25 January 2014; Accepted 2 February 2014; Published 24 March 2014

Academic Editor: Michael Meylan

Copyright (c) 2014 A. R. Seadawy et al. This is an open access article distributed under the Creative Commons Attribution License, which permits unrestricted use, distribution, and reproduction in any medium, provided the original work is properly cited.

\begin{abstract}
The Olver equation is governing a unidirectional model for describing long and small amplitude waves in shallow water waves. The solitary wave solutions of the Olver and fifth-order KdV equations can be obtained by using extended tanh and sech-tanh methods. The present results are describing the generation and evolution of such waves, their interactions, and their stability. Moreover, the methods can be applied to a wide class of nonlinear evolution equations. All solutions are exact and stable and have applications in physics.
\end{abstract}

\section{Introduction}

The research on the Korteweg-de Vries (KdV) equations attracted the interest of many scientists. The KdV equations describe nonlinear dispersive long waves; many other partial differential equations have been derived to model wave phenomena in diverse nonlinear systems. The KdV equation plays an important role in describing motions of long waves in shallow water under gravity, one-dimensional nonlinear lattice $[1,2]$, fluid mechanics $[3,4]$, quantum mechanics, plasma physics, nonlinear optics, and other areas. The KdV equation is a well-known model for the description of nonlinear long internal waves in a fluid stratified by both density and current. The steady-state version of this equation was produced by Long [5], while Benney [6] gave the integral expressions for calculation of the coefficients of the KdV equation for waves in a fluid with arbitrary stratification in the density and current.

There are many classical methods proposed to solve the KdV equations, including direct integration, Lyapunov approach, Hirota's dependent variable transformation, the inverse scattering transform, and the Bäcklund transformation [7-9]. A direct algebraic approach has also been developed by Parkes and Duffy [10] in which the solutions to the particular equation are represented by an automated tanh-function method [10]. Recently, Wazwaz considered the abundant solitons solutions, compactons and solitary patterns solutions, some new solitons, and periodic solutions of the fifth-order KdV equation [11, 12]. The adiabatic parameter dynamics of 1-soliton solution of the generalized fifth-order nonlinear KdV equation is obtained by virtue of the soliton perturbation theory $[13,14]$. The authors present a Mathematica package that deals with complicated algebraic system and outputs directly the required solutions for particular nonlinear equations [15-21].

Exact solutions to nonlinear evolution equations (NEEs) play an important role in nonlinear physical science, since the characteristics of these solutions may well simulate reallife physical phenomena [22-24]. The wave phenomena can be observed in fluid dynamics, plasma physics, elastic media, and so forth. The main task of this work is to show that our proposed methods, improved tanh and sech-tanh methods, are very efficient in solving the Olver equation and the fifthorder KdV equation by using extended tanh method and extended sech-tanh method [25-28].

This paper is organized as follows. An introduction in is presented in Section 1. In Section 2, an analysis of the extended tanh method and extended sech-tanh method is 
formulated. In Section 3, the travelling wave solutions of the Olver and the fifth-order KdV equations are obtained. Finally, the paper ends with a conclusion in Section 4.

\section{An Analysis of the Methods}

2.1. The sech-tanh Method. We suppose that $u(x, t)=u(\xi)$, where $\xi=x-k t$, and $u(\xi)$ has the following formal travelling wave solution:

$$
u(\xi)=\sum_{i=1}^{n} \operatorname{sech}^{i-1} \xi\left(A_{i} \operatorname{sech} \xi+B_{i} \tanh \xi\right),
$$

where $A_{0}, A_{1}, \ldots, A_{n}$ and $B_{1}, \ldots, B_{n}$ are constants to be determined.

Step 1. Equating the highest-order nonlinear term and the highest-order linear partial derivative in the ordinary differential equations yields the value of $n$.

Step 2. By setting the coefficients of $\operatorname{sech}^{j} \tanh ^{i}$ for $i=0,1$ and $j=1,2, \ldots$ to zero, we have the following set of over determined equations in the unknowns $A_{0}, A_{i}, B_{i}$, and $\mu$ and $k$ for $i=1,2, \ldots, n$.

Step 3. By using Mathematica and Wu's elimination methods, the algebraic equations in Step 2 can be solved.

2.2. The Extended tanh Method. The tanh method developed and introduced an independent variable:

$$
Y=\tanh (\mu \xi), \quad \xi=x-k t,
$$

that is introduced and leads to the change of the following derivatives:

$$
\begin{gathered}
\frac{d}{d \xi}=\mu\left(1-Y^{2}\right) \frac{d}{d Y} \\
\frac{d^{2}}{d \xi^{2}}=-2 \mu^{2} Y\left(1-Y^{2}\right)\left(\frac{d}{d Y}\right)^{2}+\mu^{2}\left(1-Y^{2}\right)^{2} \frac{d^{2}}{d Y^{2}} .
\end{gathered}
$$

The extended tanh method admits the use of the finite expansion:

$$
u(\mu \xi)=S(Y)=\sum_{i=0}^{m} a_{i} Y^{i}+\sum_{i=1}^{m} b_{i} Y^{-i}
$$

where $m$ is a positive integer, in most cases, that will be determined. Expansion equation (4) reduces to the standard tanh method for $b_{i}, 1 \leqslant i \leqslant m$. The parameter $m$ is usually obtained by balancing the linear terms of highest-order in the resulting equation with the highest-order nonlinear terms. Substituting of (4) into the ODE results in an algebraic system of equations in powers of $Y$ that will lead to the determination of the parameters $a_{i},(i=0, \ldots, m), \mu$ and $c$.

Stability of Solution. Hamiltonian system for the momentum is given by

$$
\nu=\frac{1}{2} \int_{-\infty}^{\infty} \int_{-\infty}^{\infty} u^{2}(x, y) d x d y, \quad \text { at } t=0 .
$$

The sufficient condition for discussing the stability of solution is $\partial v / \partial k>0$, where $k$ is coefficient of time.

\section{Application of the Methods}

3.1. The Olver Equation. In this section, we will employ the proposed methods to solve Olver equation [23]:

$$
\begin{aligned}
& v_{t}+\left(1-\frac{q_{6}^{2}}{4 q_{2}}\right) v_{x}+q_{1} v_{x x x x x}+q_{2} v^{2} v_{x}+q_{3} v v_{x x x} \\
& +q_{4} v_{x} v_{x x}+\left(q_{5}-\frac{q_{3} q_{6}}{2 q_{2}}\right) v_{x x x}=0,
\end{aligned}
$$

where the coefficients $q_{i},(i=1, \ldots, 6)$ are real constants depending on surface tension. These coefficients are

$$
\begin{gathered}
q_{1}=\left(\frac{19}{360}-\frac{\tau}{12}-\frac{\tau^{2}}{8}\right) \zeta^{2}, \quad q_{2}=-\frac{3}{8} \chi^{2}, \\
q_{3}=\left(\frac{5}{12}-\frac{\tau}{4}\right) \chi \zeta, \quad q_{4}=\left(\frac{23}{24}+\frac{5 \tau}{8}\right) \chi \zeta, \\
q_{5}=\left(\frac{1}{6}-\frac{\tau}{2}\right) \zeta, \quad q_{6}=\frac{3}{2} \chi .
\end{gathered}
$$

Here, $\tau$ represents a dimensionless surface tension coefficient, $\chi$ is the ratio of wave amplitude to undisturbed fluid depth, and $\zeta$ is the square of the ratio of fluid depth to wave length.

3.1.1. Using a sech-tanh Method. Equation (6) was equivalently

$$
\begin{aligned}
& \left(1-k-\frac{q_{6}^{2}}{4 q_{2}}\right) v^{\prime}+q_{1} v^{\prime \prime \prime \prime \prime}+q_{2} v^{2} v^{\prime}+q_{3} v v^{\prime \prime \prime} \\
& +q_{4} v^{\prime} v^{\prime \prime}+\left(q_{5}-\frac{q_{3} q_{6}}{2 q_{2}}\right) v^{\prime \prime \prime}=0
\end{aligned}
$$

obtained upon using the wave variable $\xi=x-k t$. Balancing $v^{\prime \prime \prime \prime \prime \prime}$ with $v^{\prime} v^{\prime \prime}$ in (8) gives $m=2$. sech-tanh method equation (1) admits the use of the finite expansion:

$$
\begin{aligned}
v(\xi)= & A_{0}+A_{1} \operatorname{sech} \xi+B_{1} \tanh \xi \\
& +A_{2} \operatorname{sech}^{2} \xi+B_{2} \operatorname{sech} \xi \tanh \xi
\end{aligned}
$$

and by substituting from (9) into (8) and setting the coefficients of $\operatorname{sech}^{j} \tanh ^{i}$ for $i=0,1$ and $j=1,2,3,4,5,6,7$ to zero, we have the following set of overdetermined equations in the unknowns $A_{0}, A_{1}, A_{2}, B_{1}, B_{2}$, and $k$.

By solving the set of result equations by using Mathematica, we obtain the following solutions. 
Case I. Consider

$$
\begin{gathered}
A_{1}=B_{1}=B_{2}=0, \quad A_{2}=\frac{3\left(2 q_{3}+q_{4} \mp E\right)}{q_{2}} \\
A_{0}=\frac{1}{4 q_{2}\left(10 q_{1} q_{2}-q_{3}\left(q_{3}+q_{4}\right)\right)} \\
\times\left(8 q_{3}^{3}+4 q_{3}^{2}\left(3 q_{4} \mp E\right)\right. \\
+40 q_{1} q_{2}\left(-2 q_{3}-q_{4} \pm \mathrm{E}\right) \\
+2 q_{2} q_{5}\left(q_{4} \pm E\right) \\
\left.+q_{3}\left(4 q_{4}^{2} \mp E q_{6}-q_{4}\left(q_{6} \pm 4 E\right)\right)\right) \\
+\frac{1}{8 q_{2}\left(-10 q_{1} q_{2}+q_{3}\left(q_{3}+q_{4}\right)\right)^{2}} \\
\times\left(-19200 q_{1}^{3} q_{2}^{3}\right. \\
+4 q_{2}^{2} q_{5}^{2}\left(2 q_{3}^{2}+2 q_{3} q_{4}+q_{4}\left(q_{4} \pm E\right)\right) \\
+q_{3}^{2} q_{4}\left(2 q_{3}+q_{4} \mp E\right)\left(4\left(q_{3}+q_{4}\right)-q_{6}\right) \\
\times\left(4\left(q_{3}+q_{4}\right)+q_{6}\right) \\
+40 q_{3}^{3}+256 q_{3}^{2} q_{4}+q_{3}\left(288 q_{4}^{2} \mp 80 q_{4} E-5 q_{6}^{2}\right) \\
+4 q_{2} q_{3}\left(2 q_{3}\left(2 q_{3}\left(q_{3}+q_{4} q_{4}\left(q_{4} \mp E\right)-q_{6}^{2}\right)\right)\right) \\
+4 q_{1} q_{2}\left(20 q_{2}^{2} q_{5}^{2}\right. \\
\left.+2 q_{3}^{2}+2 q_{3} q_{4}+q_{4}^{2}\right) q_{5} q_{6}
\end{gathered}
$$

In this case, the generalized soliton solution can be written as

$$
\begin{aligned}
v_{1}(x, t)= & \frac{1}{4 q_{2}\left(10 q_{1} q_{2}-q_{3}\left(q_{3}+q_{4}\right)\right)} \\
& \times\left(8 q_{3}^{3}+4 q_{3}^{2}\left(3 q_{4} \mp E\right)\right. \\
& \quad+40 q_{1} q_{2}\left(-2 q_{3}-q_{4} \pm E\right)+2 q_{2} q_{5}\left(q_{4} \pm E\right) \\
& \left.\quad+q_{3}\left(4 q_{4}^{2} \mp E q_{6}-q_{4}\left(q_{6} \pm 4 E\right)\right)\right) \\
+ & \left(\frac{3\left(2 q_{3}+q_{4} \mp E\right)}{q_{2}}\right) \operatorname{sech}^{2}[x-k t],
\end{aligned}
$$

where $E=\sqrt{-40 q_{1} q_{2}+\left(2 q_{3}+q_{4}\right)^{2}}$, and $\left(2 q_{3}+q_{4}\right)^{2}>40 q_{1} q_{2}$.

Figure 1(a) shows the stability dark solitary wave solutions with $(\chi=0.25, \zeta=0.025$, and $\tau=0.5)$ in the interval
$[-10,10]$ and time in the interval $[0,5]$. Figure $1(b)$ shows the stability contour of solitary wave solution with $(\chi=0.25$, $\zeta=0.025$, and $\tau=0.5)$ in the interval $[-10,10]$ and time in the interval $[0,5]$.

Case II. Consider

$$
\begin{aligned}
& A_{0}=\frac{1}{4 q_{2}\left(10 q_{1} q_{2}-q_{3}\left(q_{3}+q_{4}\right)\right)} \\
& \cdot\left(2 q_{3}^{3}-10 q_{1} q_{2}\left(E+2 q_{3}+q_{4}\right)+q_{3}^{2}\left(E+3 q_{4}\right)\right. \\
& +2 q_{2}\left(-E+q_{4}\right) q_{5} \\
& \left.+q_{3}\left(q_{4}\left(E+q_{4}\right)+\left(E-q_{4}\right) q_{6}\right)\right), \\
& A_{1}=B_{1}=0, \quad A_{2}=\frac{3\left(2 q_{3}+q_{4}+E\right)}{2 q_{2}}, \\
& B_{2}=\mp \frac{3}{\sqrt{2} q_{2}} \sqrt{20 q_{1} q_{2}-\left(2 q_{3}+q_{4}\right)^{2}-\left(2 q_{3}+q_{4}\right) E} \text {, } \\
& k=\frac{1}{8 q_{2}\left(-10 q_{1} q_{2}+q_{3}\left(q_{3}+q_{4}\right)\right)^{2}} \\
& \cdot\left(-1200 q_{1}^{3} q_{2}^{3}\right. \\
& +4 q_{2}^{2} q_{5}^{2}\left(2 q_{3}^{2}-q_{4}\left(E-2 q_{3}\right)+q_{4}^{2}\right) \\
& +4 q_{2} q_{3}\left(2 q_{3}\left(q_{3}+q_{4}\right)^{2}\right. \\
& \left.-\left(2 q_{3}^{2}-q_{4}\left(E-2 q_{3}\right)+q_{4}^{2}\right) q_{5} q_{6}\right) \\
& +20 q_{1}^{2} q_{2}^{2}\left(5 q_{4}\left(E+q_{4}\right)\right. \\
& \left.+40 q_{2}+12 q_{3}^{2}+22 q_{3} q_{4}-10 q_{6}^{2}\right) \\
& +q_{3}^{2} q_{4}\left(E+2 q_{3}+q_{4}\right)\left(\left(q_{3}+q_{4}\right)^{2}-q_{6}^{2}\right) \\
& -4 q_{1} q_{2}\left(20 q_{2}^{2} q_{5}^{2}+20 q_{2} q_{3}\left(\left(2 q_{3}+q_{4}\right)-q_{5} q_{6}\right)\right. \\
& +q_{3}\left(\left(q_{3}+q_{4}\right)\right. \\
& \times\left(3 q_{3}^{2}+13 q_{3} q_{4}+5 q_{4}\left(E+q_{4}\right)\right) \\
& \left.\left.\left.-5 q_{6}^{2}\left(q_{3}+2 q_{4}\right)\right)\right)\right) \text {. }
\end{aligned}
$$

In this case, the generalized soliton solution can be written as

$$
\begin{aligned}
v_{2}(x, t)= & \frac{1}{4 q_{2}\left(10 q_{1} q_{2}-q_{3}\left(q_{3}+q_{4}\right)\right)} \\
& \cdot\left(2 q_{3}^{3}-10 q_{1} q_{2}\left(E+2 q_{3}+q_{4}\right)+q_{3}^{2}\left(E+3 q_{4}\right)\right. \\
& +2 q_{2}\left(-E+q_{4}\right) q_{5} \\
& \left.+q_{3}\left(q_{4}\left(E+q_{4}\right)+\left(E-q_{4}\right) q_{6}\right)\right) \\
& +\left(\frac{3\left(2 q_{3}+q_{4}+E\right)}{2 q_{2}}\right) \operatorname{sech}^{2}[x-k t] \\
& \mp\left(\frac{3}{\sqrt{2} q_{2}} \sqrt{20 q_{1} q_{2}-\left(2 q_{3}+q_{4}\right)^{2}-\left(2 q_{3}+q_{4}\right) E}\right) \\
& \times \operatorname{sech}[x-k t] \tanh [x-k t] .
\end{aligned}
$$




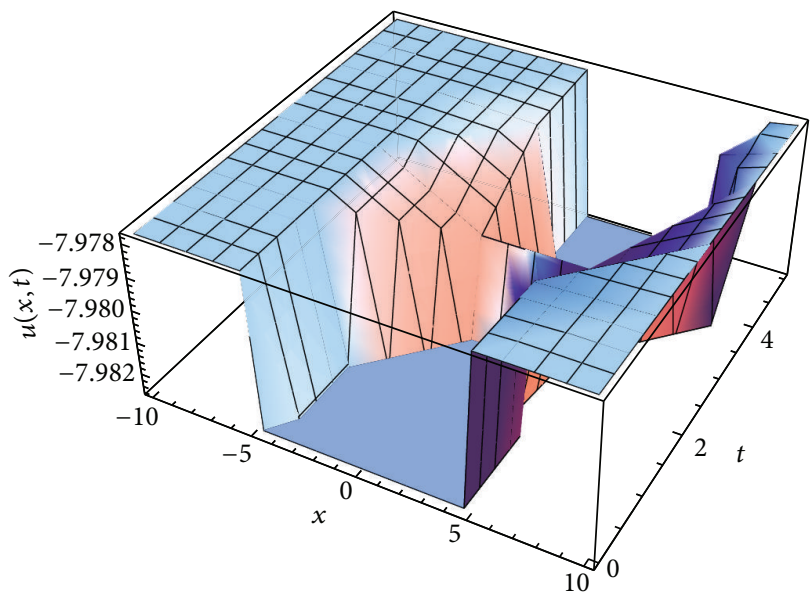

(a)

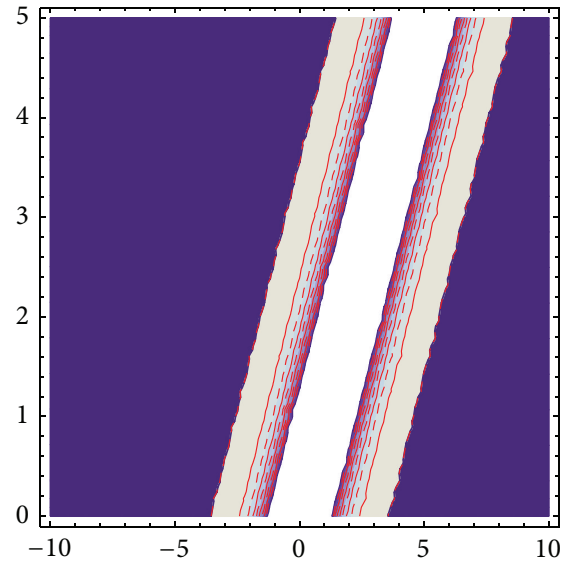

(b)

FIGURE 1: (a) Travelling waves solutions of (11) is plotted: stability dark solitary waves. (b) Travelling waves solutions of (11) is plotted: stability contour of solitary waves.

Case III. Consider

$$
\begin{aligned}
& A_{0}=\frac{1}{4 q_{2}\left(10 q_{1} q_{2}-q_{3}\left(q_{3}+q_{4}\right)\right)} \\
& \cdot\left(2 q_{3}^{3}-10 q_{1} q_{2}\left(-E+2 q_{3}+q_{4}\right)\right. \\
& +q_{3}^{2}\left(-E+3 q_{4}\right)+2 q_{2}\left(E+q_{4}\right) q_{5} \\
& \left.+q_{3}\left(q_{4}\left(-E+q_{4}\right)-\left(E+q_{4}\right) q_{6}\right)\right) \text {, } \\
& A_{1}=B_{1}=0, \quad A_{2}=\frac{3\left(2 q_{3}+q_{4}-E\right)}{2 q_{2}}, \\
& B_{2}=\mp \frac{3}{\sqrt{2} q_{2}} \sqrt{20 q_{1} q_{2}-\left(2 q_{3}+q_{4}\right)^{2}+\left(2 q_{3}+q_{4}\right) E}, \\
& k=\frac{1}{\left(8 q_{2}-10 q_{1} q_{2}+q_{3}\left(q_{3}+q_{4}\right)\right)^{2}} \\
& \cdot\left(-1200 q_{1}^{3} q_{2}^{3}\right. \\
& +4 q_{2}^{2} q_{5}^{2}\left(2 q_{3}^{2}+q_{4}\left(E+2 q_{3}\right)+q_{4}^{2}\right) \\
& +4 q_{2} q_{3}\left(2 q_{3}\left(q_{3}+q_{4}\right)^{2}\right. \\
& \left.-\left(2 q_{3}^{2}+q_{4}\left(E+2 q_{3}\right)+q_{4}^{2}\right) q_{5} q_{6}\right) \\
& +20 q_{1}^{2} q_{2}^{2}\left(5 q_{4}\left(-E+q_{4}\right)+40 q_{2}\right. \\
& \left.+12 q_{3}^{2}+22 q_{3} q_{4}-10 q_{6}^{2}\right) \\
& +q_{3}^{2} q_{4}\left(-E+2 q_{3}+q_{4}\right)\left(\left(q_{3}+q_{4}\right)^{2}-q_{6}^{2}\right) \\
& -4 q_{1} q_{2}\left(20 q_{2}^{2} q_{5}^{2}+20 q_{2} q_{3}\left(\left(2 q_{3}+q_{4}\right)-q_{5} q_{6}\right)\right. \\
& +q_{3}\left(\left(q_{3}+q_{4}\right)\right. \\
& \times\left(3 q_{3}^{2}+13 q_{3} q_{4}+5 q_{4}\left(-E+q_{4}\right)\right) \\
& \left.\left.\left.-5 q_{6}^{2}\left(q_{3}+2 q_{4}\right)\right)\right)\right) \text {. }
\end{aligned}
$$

In this case, the generalized soliton solution can be written as

$$
\begin{aligned}
v_{3}(x, t)= & \frac{1}{4 q_{2}\left(10 q_{1} q_{2}-q_{3}\left(q_{3}+q_{4}\right)\right)} \\
& \cdot\left(2 q_{3}^{3}-10 q_{1} q_{2}\left(-E+2 q_{3}+q_{4}\right)+q_{3}^{2}\left(-E+3 q_{4}\right)\right. \\
& +2 q_{2}\left(E+q_{4}\right) q_{5}+q_{3}\left(q_{4}\left(-E+q_{4}\right)\right. \\
& +\left(\frac{3\left(2 q_{3}+q_{4}-E\right)}{2 q_{2}}\right) \operatorname{sech}^{2}[x-k t] \\
& \mp\left(\frac{3}{\sqrt{2} q_{2}} \sqrt{20 q_{1} q_{2}-\left(2 q_{3}+q_{4}\right)^{2}+\left(2 q_{3}+q_{4}\right) E}\right) \\
& \times \operatorname{sech}[x-k t] \tanh [x-k t] .
\end{aligned}
$$

3.1.2. Using the Extended tanh Method. We have

$$
u(\xi)=a_{0}+a_{1} Y+a_{2} Y^{2}+\frac{b_{1}}{Y}+\frac{b_{2}}{Y^{2}} .
$$

By substituting (16) into (8) and collecting the coefficient of $Y$, we obtain a system of algebraic equations for $a_{0}, a_{1}, a_{2}, b_{1}$, $b_{2}$, and $k$. Solving this system gives the following solution.

Case I. Consider

$$
\begin{gathered}
a_{1}=b_{1}=b_{2}=0, \quad a_{2}=-\frac{3 \mu^{2}\left(2 q_{3}+q_{4} \pm E\right)}{q_{2}}, \\
a_{0}=\frac{1}{q_{2}\left(q_{4} \pm E\right)} \cdot\left(4 \mu^{2}\left(-20 q_{1} q_{2}+\left(q_{3}+q_{4}\right)\left(2 q_{3}+q_{4}\right)\right)\right. \\
\left. \pm 4 \mu^{2} E\left(q_{3}+q_{4}\right)+2 q_{2} q_{5}-q_{3} q_{6}\right),
\end{gathered}
$$




$$
\begin{gathered}
k=\frac{1}{8 q_{2}\left(-10 q_{1} q_{2}+q_{3}\left(q_{3}+q_{4}\right)\right)^{2}} \\
\cdot\left(-19200 \mu^{4} q_{1}^{3} q_{2}^{3}\right. \\
+4 q_{2}^{2} q_{5}^{2}\left(2 q_{3}^{2}-\left( \pm E-2 q_{3}\right) q_{4}+q_{4}^{2}\right) \\
+4 q_{2} q_{3}\left(2 q_{3}\left(q_{3}+q_{4}\right)^{2}\right. \\
\left.-\left(2 q_{3}^{2}-\left( \pm E-2 q_{3}\right) q_{4}+q_{4}^{2}\right) q_{5} q_{6}\right) \\
+40 q_{1}^{2} q_{2}^{2}\left(20 q_{2}+8 \mu^{4}\left(12 q_{3}^{2}+22 q_{3} q_{4}\right.\right. \\
\left.+q_{3}^{2} q_{4}\left( \pm E q_{4}\left( \pm E+q_{4}\right)\right)-5 q_{6}^{2}\right) \\
-4 q_{1} q_{2}\left(8 q_{3}\left(q_{3}+q_{4}\right)\right. \\
\quad \times\left(5 q_{2}+2 \mu^{4}\right)\left(16 \mu^{4}\left(q_{3}+q_{4}\right)^{2}-q_{6}^{2}\right) \\
\left.\quad \times\left(3 q_{3}^{2}+13 q_{3} q_{4}+5 q_{4}\left( \pm E+q_{4}\right)\right)\right) \\
+20 q_{2}^{2} q_{5}^{2}-20 q_{2} q_{3} q_{5} q_{6} \\
\left.\left.-5 q_{3}\left(q_{3}+2 q_{4}\right) q_{6}^{2}\right)\right) .
\end{gathered}
$$

In this case, the generalized soliton solution can be written as

$$
\begin{aligned}
u_{1}(x, t)= & \frac{1}{q_{2}\left(q_{4} \pm E\right)} \\
& \cdot\left(4 \mu ^ { 2 } \left(-20 q_{1} q_{2}\right.\right. \\
& \left.\quad+\left(q_{3}+q_{4}\right)\left(2 q_{3}+q_{4}\right)\right) \\
& \left. \pm 4 \mu^{2} E\left(q_{3}+q_{4}\right)+2 q_{2} q_{5}-q_{3} q_{6}\right) \\
- & \frac{3 \mu^{2}\left(2 q_{3}+q_{4} \pm E\right)}{q_{2}} \tanh ^{2}[x-k t],
\end{aligned}
$$

where $E=\sqrt{-40 q_{1} q_{2}+\left(2 q_{3}+q_{4}\right)^{2}}$, and $\left(2 q_{3}+q_{4}\right)^{2}>40 q_{1} q_{2}$.

Figure 2(a) shows the stability dark solitary wave solutions with $(\chi=0.25, \zeta=0.025$, and $\tau=0.5)$ in the interval $[-10,10]$ and time in the interval $[0,5]$. Figure $2(b)$ shows the stability contour of solitary wave solution with $(\chi=0.25$, $\zeta=0.025$, and $\tau=0.5)$ in the interval $[-10,10]$ and time in the interval $[0,5]$.

Case II. Consider

$$
\begin{aligned}
a_{1}=b_{1}= & 0, \quad a_{2}=b_{2}=-\frac{3 \mu^{2}\left(2 q_{3}+q_{4} \pm E\right)}{q_{2}}, \\
a_{0}= & \frac{1}{q_{2}\left(q_{4} \pm E\right)} \\
& \cdot\left(4 \mu^{2}\left(-20 q_{1} q_{2}+\left(q_{3}+q_{4}\right)\left(2 q_{3}+q_{4}\right)\right)\right. \\
& \left. \pm 4 \mu^{2} E\left(q_{3}+q_{4}\right)+2 q_{2} q_{5}-q_{3} q_{6}\right),
\end{aligned}
$$

$$
\begin{gathered}
k=\frac{1}{8 q_{2}\left(-10 q_{1} q_{2}+q_{3}\left(q_{3}+q_{4}\right)\right)^{2}} \\
\cdot\left(-307200 \mu^{4} q_{1}^{3} q_{2}^{3}\right. \\
+4 q_{2}^{2} q_{5}^{2}\left(2 q_{3}^{2}-\left( \pm E-2 q_{3}\right) q_{4}+q_{4}^{2}\right) \\
+4 q_{2} q_{3}\left(2 q_{3}\left(q_{3}+q_{4}\right)^{2}\right. \\
\left.-\left(2 q_{3}^{2}-\left( \pm E-2 q_{3}\right) q_{4}+q_{4}^{2}\right) q_{5} q_{6}\right) \\
+40 q_{1}^{2} q_{2}^{2}\left(20 q_{2}\right. \\
+128 \mu^{4}\left(12 q_{3}^{2}+22 q_{3} q_{4}\right. \\
\left.\left.+5 q_{4}\left( \pm E+q_{4}\right)\right)-5 q_{6}^{2}\right) \\
+q_{3}^{2} q_{4}\left( \pm E+2 q_{3}+q_{4}\right)\left(256 \mu^{4}\left(q_{3}+q_{4}\right)^{2}-q_{6}^{2}\right) \\
-4 q_{1} q_{2}\left(8 q_{3}\left(q_{3}+q_{4}\right)\right. \\
\times\left(5 q_{2}+32 \mu^{4}\right. \\
\left.\times\left(3 q_{3}^{2}+13 q_{3} q_{4}+5 q_{4}\left( \pm E+q_{4}\right)\right)\right) \\
+20 q_{2}^{2} q_{5}^{2}-20 q_{2} q_{3} q_{5} q_{6} \\
\left.\left.-5 q_{3}\left(q_{3}+2 q_{4}\right) q_{6}^{2}\right)\right) .
\end{gathered}
$$

In this case, the generalized soliton solution can be written as

$$
\begin{aligned}
u_{2}(x, t)= & \frac{1}{q_{2}\left(q_{4} \pm E\right)} \\
& \cdot\left(4 \mu ^ { 2 } \left(-20 q_{1} q_{2}\right.\right. \\
& \left.\quad+\left(q_{3}+q_{4}\right)\left(2 q_{3}+q_{4}\right)\right) \\
& \left. \pm 4 \mu^{2} E\left(q_{3}+q_{4}\right)+2 q_{2} q_{5}-q_{3} q_{6}\right) \\
- & \frac{3 \mu^{2}\left(2 q_{3}+q_{4} \pm E\right)}{q_{2}} \\
& \times\left(\tanh ^{2}[x-k t]+\operatorname{coth}^{2}[x-k t]\right) .
\end{aligned}
$$

Case III. Consider

$$
\begin{gathered}
a_{1}=a_{2}=b_{1}=0, \quad b_{2}=-\frac{3 \mu^{2}\left(2 q_{3}+q_{4} \pm E\right)}{q_{2}}, \\
a_{0}=\frac{1}{q_{2}\left(q_{4} \pm E\right)} \cdot\left(4 \mu^{2}\left(-20 q_{1} q_{2}+\left(q_{3}+q_{4}\right)\left(2 q_{3}+q_{4}\right)\right)\right. \\
\left. \pm 4 \mu^{2} E\left(q_{3}+q_{4}\right)+2 q_{2} q_{5}-q_{3} q_{6}\right),
\end{gathered}
$$




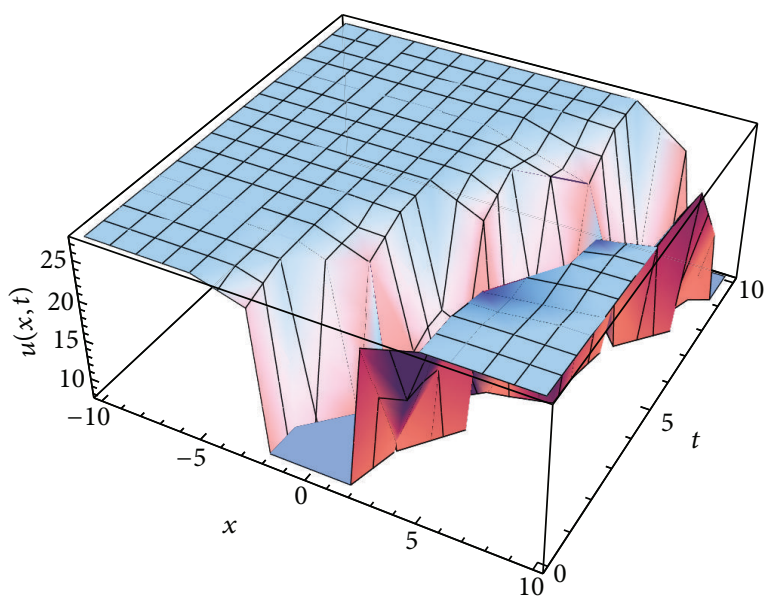

(a)

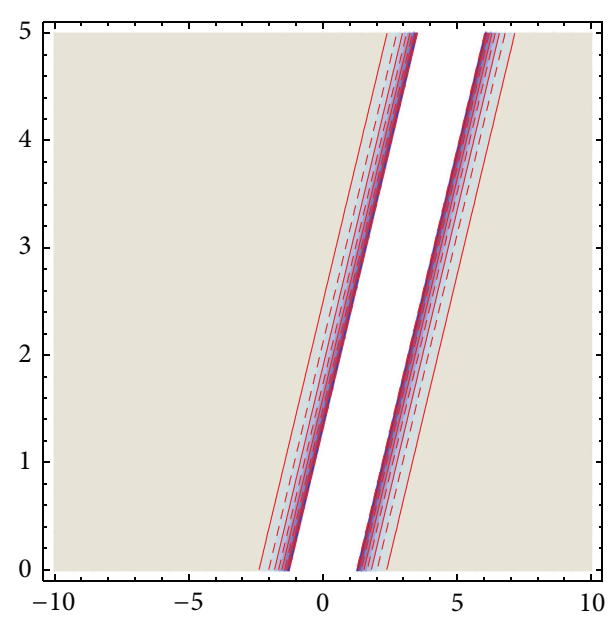

(b)

FIGURE 2: (a) Travelling waves solutions of (18) is plotted: stability dark solitary waves. (b) Travelling waves solutions of (18) is plotted: stability contour of solitary waves.

$$
\begin{gathered}
k=\frac{1}{8 q_{2}\left(-10 q_{1} q_{2}+q_{3}\left(q_{3}+q_{4}\right)\right)^{2}} \\
\cdot\left(-19200 \mu^{4} q_{1}^{3} q_{2}^{3}\right. \\
+4 q_{2}^{2} q_{5}^{2}\left(2 q_{3}^{2}-\left( \pm E-2 q_{3}\right) q_{4}+q_{4}^{2}\right) \\
+4 q_{2} q_{3}\left(2 q_{3}\left(q_{3}+q_{4}\right)^{2}\right. \\
\left.-\left(2 q_{3}^{2}-\left( \pm E-2 q_{3}\right) q_{4}+q_{4}^{2}\right) q_{5} q_{6}\right) \\
+40 q_{1}^{2} q_{2}^{2}\left(20 q_{2}\right. \\
+8 \mu^{4}\left(12 q_{3}^{2}+22 q_{3} q_{4}\right. \\
\left.+q_{3}^{2} q_{4}\left( \pm E q_{4}\left( \pm E+q_{4}\right)\right)-5 q_{6}^{2}\right) \\
-4 q_{1} q_{2}\left(8 q_{3}\left(q_{3}+q_{4}\right)\right. \\
\times\left(5 q_{2}+2 \mu^{4}\right. \\
\left.\times\left(3 q_{3}^{2}+13 q_{3} q_{4}+5 q_{4}\left( \pm E+q_{4}\right)\right)\right) \\
+20 q_{2}^{2} q_{5}^{2}-20 q_{2} q_{3} q_{5} q_{6} \\
\left.\left.\left.\left.-5 q_{3}\left(q_{3}+2 q_{4}\right) q_{6}^{2}\right)\right) . q_{4}\right)^{2}-q_{6}^{2}\right) \\
\times
\end{gathered}
$$

In this case, the generalized soliton solution can be written as

$$
\begin{aligned}
u_{3}(x, t)= & \frac{1}{q_{2}\left(q_{4} \pm E\right)} \\
& \cdot\left(4 \mu ^ { 2 } \left(-20 q_{1} q_{2}\right.\right. \\
& \left.+\left(q_{3}+q_{4}\right)\left(2 q_{3}+q_{4}\right)\right) \pm 4 \mu^{2} E\left(q_{3}+q_{4}\right) \\
& \left.+2 q_{2} q_{5}-q_{3} q_{6}\right) \\
& -\frac{3 \mu^{2}\left(2 q_{3}+q_{4} \pm E\right)}{q_{2}} \operatorname{coth}^{2}[x-k t] .
\end{aligned}
$$

3.2. Solving the Fifth-Order Korteweg-de Vries Equation. In this section we will employ the proposed methods to solve the fifth-order Korteweg-de Vries equation:

$$
\begin{gathered}
\eta_{t}+6 \eta \eta_{x}+\eta_{3 x}+\alpha c_{1} \eta^{2} \eta_{x}+\alpha c_{2} \eta_{x} \eta_{x x} \\
+\alpha c_{3} \eta \eta_{3 x}+\alpha c_{4} \eta_{5 x}=0, \quad \alpha \ll 1 .
\end{gathered}
$$

Or equivalently

$$
\begin{gathered}
-k \eta^{\prime}+6 \eta \eta^{\prime}+\eta^{\prime \prime \prime}+\alpha c_{1} \eta^{2} \eta^{\prime}+\alpha c_{2} \eta^{\prime} \eta^{\prime \prime} \\
+\alpha c_{3} \eta \eta^{\prime \prime \prime}+\alpha c_{4} \eta^{\prime \prime \prime \prime \prime \prime}=0,
\end{gathered}
$$

is obtained upon using the wave variable $\xi=x-k t$, when the higher-order coefficients are given by

$$
\left(c_{1}, c_{2}, c_{3}, c_{4}\right)=\left(1, \frac{1}{12}, \frac{1}{3}, \frac{1}{480}\right)
$$

3.2.1. Using a sech-tanh Method. Balancing $\eta^{\prime \prime \prime \prime \prime}$ with $\eta \eta^{\prime \prime \prime}$ in (24) gives $m=2$. sech-tanh method equation (1) admits the use of the finite expansion:

$$
\begin{aligned}
\eta(\xi)= & A_{0}+A_{1} \operatorname{sech} \xi+B_{1} \tanh \xi \\
& +A_{2} \operatorname{sech}^{2} \xi+B_{2} \operatorname{sech} \xi \tanh \xi
\end{aligned}
$$

and by substituting from (27) into (24) and setting the coefficients of $\operatorname{sech}^{j} \tanh ^{i}$ for $i=0,1$ and $j=1,2,3,4,5,6,7$ to zero, we have the following set of overdetermined equations in the unknowns $A_{0}, A_{1}, A_{2}, B_{1}, B_{2}$, and $k$.

Solve the set of result equations by using Mathematica; we obtain the following solutions. 
Case I. Consider

$$
\begin{gathered}
A_{1}=B_{1}=B_{2}=0, \quad A_{2}=\frac{3 c_{2}+6 c_{3} \mp 3 g}{c_{1}}, \\
A_{0}=\frac{1}{2 \alpha c_{1}\left(-c_{3}\left(c_{2}+c_{3}\right)+10 c_{1} c_{4}\right)} \\
\cdot\left(c_{3}\left(3+2 \alpha\left(c_{2}+c_{3}\right)\right)\left(c_{2}+2 c_{3} \mp g\right)\right. \\
+c_{1}\left(c_{2}\left(1-20 \alpha c_{4}\right) \pm g\right. \\
\left.\left.+20 c_{4}\left(-3-2 \alpha c_{3} \pm \alpha g\right)\right)\right) \\
\frac{-1}{2 \alpha c_{1}\left(c_{3}\left(c_{2}+c_{3}\right)-10 c_{1} c_{4}\right)^{2}} \\
\cdot\left(20 c_{1}^{3}\left(c_{4}+240 \alpha^{2} c_{4}^{3}\right)\right. \\
+c_{2} c_{3}^{2}\left(-3+2 \alpha\left(c_{2}+c_{3}\right)\right) \\
\times\left(3+2 \alpha\left(c_{2}+c_{3}\right)\right)\left(-c_{2}-2 c_{3} \pm g\right) \\
-c_{1}^{2}\left(c_{2}^{2}\left(1+400 \alpha^{2} c_{4}^{2}\right)\right. \\
+2\left(c_{3}^{2}+60 c_{3} c_{4}+60\left(-15+8 \alpha^{2} c_{3}^{2}\right) c_{4}^{2}\right) \\
\left.+c_{2}\left( \pm g\left(1-400 \alpha^{2} c_{4}^{2}\right)+2 c_{3}\left(1+880 \alpha^{2} c_{4}^{2}\right)\right)\right) \\
+2 c_{1} c_{3}\left(40 \alpha^{2} c_{2}^{3} c_{4}+6 c_{3}\left(c_{3}+\left(-15+4 \alpha^{2} c_{3}^{2}\right) c_{4}\right)\right. \\
+c_{2}^{2}\left(3+8 \alpha^{2} c_{4}\left(18 c_{3} \mp 5 g\right)\right) \\
+c_{2}\left(3\left(-60 c_{4} \pm g\right)+2 c_{3}\right. \\
\left.\left.\times\left(3 \alpha^{2} c_{4}\left(16 c_{3} \mp 5 g\right)\right)\right)\right) .
\end{gathered}
$$

In this case, the generalized soliton solution can be written as

$$
\begin{aligned}
\eta_{1}(x, t)= & \frac{1}{2 \alpha c_{1}\left(-c_{3}\left(c_{2}+c_{3}\right)+10 c_{1} c_{4}\right)} \\
& \cdot\left(c_{3}\left(3+2 \alpha\left(c_{2}+c_{3}\right)\right)\left(c_{2}+2 c_{3} \mp g\right)\right. \\
& +c_{1}\left(c_{2}\left(1-20 \alpha c_{4}\right) \pm g\right. \\
& \left.\left.+20 c_{4}\left(-3-2 \alpha c_{3} \pm \alpha g\right)\right)\right) \\
& +\frac{3 c_{2}+6 c_{3} \mp 3 g}{c_{1}} \operatorname{sech}^{2}[x-k t]
\end{aligned}
$$

where $g=\sqrt{\left(c_{2}+2 c_{3}\right)^{2}-40 c_{1} c_{4}}$ and $\left(c_{2}+2 c_{3}\right)^{2}>40 c_{1} c_{4}$.

Figure 3(a) shows the stability bright solitary wave solutions with $(\alpha=-1)$ in the interval $[-5,5]$ and time in the interval $[0,0.5]$.

Figure $3(\mathrm{~b})$ shows the stability contour of solitary wave solution with $(\alpha=-1)$ in the interval $[-5,5]$ and time in the interval $[0,0.5]$.

Case II. Consider

$$
\begin{gathered}
A_{1}=B_{1}=0, \quad A_{2}=\frac{3\left(c_{2}+2 c_{3}+g\right)}{2 c_{1}}, \\
B_{2}=\mp \frac{3}{\sqrt{2} c_{1}} \cdot \sqrt{-\left(c_{2}+2 c_{3}\right)^{2}+20 c_{1} c_{4}-\left(c_{2}+c_{3}\right) g}
\end{gathered}
$$

$$
\begin{gathered}
A_{0}=\frac{-1}{4 \alpha c_{1}\left(c_{3}\left(c_{2}+c_{3}\right)-10 c_{1} c_{4}\right)} \\
\cdot\left(c_{3}\left(6+\alpha\left(c_{2}+c_{3}\right)\right)\left(c_{2}+2 c_{3}+g\right)\right. \\
-2 c_{1}\left(g+5\left(12+g \alpha+2 \alpha c_{3}\right) c_{4}\right. \\
\left.\left.+c_{2}\left(-1+5 \alpha c_{4}\right)\right)\right), \\
\frac{1}{8 \alpha c_{1}\left(c_{3}\left(c_{2}+c_{3}\right)-10 c_{1} c_{4}\right)^{2}} \\
+\left(-80 c_{1}^{3} c_{4}\left(1+15 \alpha^{2} c_{4}^{2}\right)\right. \\
+c_{2} c_{3}^{2}\left(-6+\alpha\left(c_{2}+c_{3}\right)\right)\left(6+\alpha\left(c_{2}+c_{3}\right)\right)\left(c_{2}+2 c_{3}+g\right) \\
-4 c_{1} c_{3}\left(5 \alpha^{2} c_{2}^{3} c_{4}+c_{2}^{2}\left(6+\alpha^{2}\left(5 g+18 c_{3}\right) c_{4}\right)\right. \\
+3 c_{3}\left(4 c_{3}+\left(-60+\alpha^{2} c_{3}^{2}\right) c_{4}\right) \\
+c_{2}\left(-6\left(g+60 c_{4}\right)\right. \\
\left.\left.+c_{3}\left(12+\alpha^{2}\left(5 g+16 c_{3}\right) c_{4}\right)\right)\right) \\
\left.\left.+c_{2}\left(g\left(-1+25 \alpha^{2} c_{4}^{2}\right)+2 c_{3}\left(1+55 \alpha^{2} c_{4}^{2}\right)\right)\right)\right) . \\
+4 c_{1}^{2}\left(c_{2}^{2}\left(1+25 \alpha^{2} c_{4}^{2}\right)\right. \\
+2\left(c_{3}^{2}+60 c_{3} c_{4}+30\left(-30+\alpha^{2} c_{3}^{2}\right) c_{4}^{2}\right)
\end{gathered}
$$

In this case, the generalized soliton solution can be written as

$$
\begin{aligned}
\eta_{2}(x, t)= & \frac{-1}{4 \alpha c_{1}\left(c_{3}\left(c_{2}+c_{3}\right)-10 c_{1} c_{4}\right)} \\
& \cdot\left(c_{3}\left(6+\alpha\left(c_{2}+c_{3}\right)\right)\left(c_{2}+2 c_{3}+g\right)\right. \\
& -2 c_{1}\left(g+5\left(12+g \alpha+2 \alpha c_{3}\right) c_{4}\right. \\
& \left.\left.+c_{2}\left(-1+5 \alpha c_{4}\right)\right)\right) \\
+ & \frac{3\left(c_{2}+2 c_{3}+g\right)}{2 c_{1}} \operatorname{sech}^{2}[x-k t] \mp \frac{3}{\sqrt{2} c_{1}} \\
& \cdot \sqrt{-\left(c_{2}+2 c_{3}\right)^{2}+20 c_{1} c_{4}-\left(c_{2}+c_{3}\right) g} \\
& \times \operatorname{sech}[x-k t] \tanh [x-k t] .
\end{aligned}
$$

Case III. Consider

$$
\begin{gathered}
A_{1}=B_{1}=0, \quad A_{2}=\frac{3\left(c_{2}+2 c_{3}-g\right)}{2 c_{1}}, \\
B_{2}= \pm \frac{3}{\sqrt{2} c_{1}} \cdot \sqrt{-\left(c_{2}+2 c_{3}\right)^{2}+20 c_{1} c_{4}+\left(c_{2}+c_{3}\right) g} \\
A_{0}=\frac{-1}{4 \alpha c_{1}\left(c_{3}\left(c_{2}+c_{3}\right)-10 c_{1} c_{4}\right)} \\
\cdot\left(c_{3}\left(6+\alpha\left(c_{2}+c_{3}\right)\right)\left(c_{2}+2 c_{3}-g\right)\right. \\
-2 c_{1}\left(-g+5\left(12-g \alpha+2 \alpha c_{3}\right) c_{4}\right. \\
\left.\left.+c_{2}\left(-1+5 \alpha c_{4}\right)\right)\right),
\end{gathered}
$$




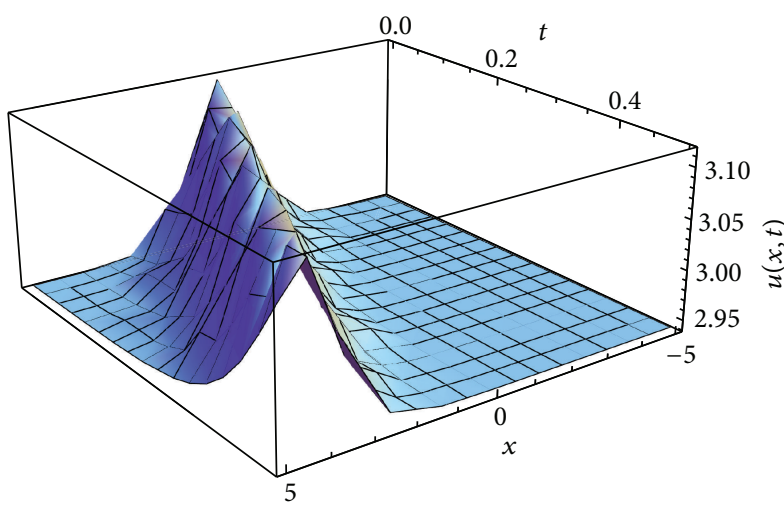

(a)

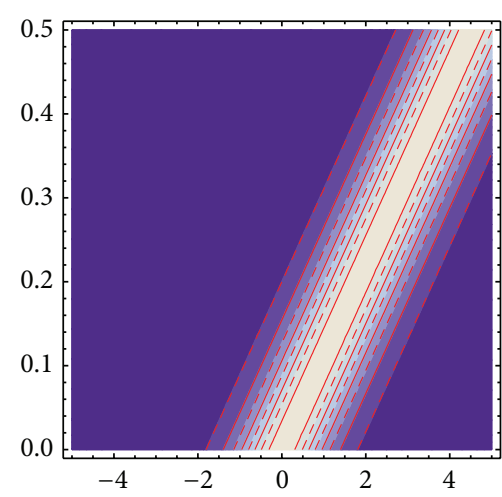

(b)

FIGURE 3: (a) Travelling waves solutions of (28) is plotted: stability bright solitary waves. (b) Travelling waves solutions of (28) is plotted: stability contour of solitary waves.

$$
\begin{gathered}
k=\frac{1}{8 \alpha c_{1}\left(c_{3}\left(c_{2}+c_{3}\right)-10 c_{1} c_{4}\right)^{2}} \\
\cdot\left(-80 c_{1}^{3} c_{4}\left(1+15 \alpha^{2} c_{4}^{2}\right)\right. \\
+c_{2} c_{3}^{2}\left(-6+\alpha\left(c_{2}+c_{3}\right)\right)\left(6+\alpha\left(c_{2}+c_{3}\right)\right) \\
\quad \times\left(c_{2}+2 c_{3}-g\right) \\
-4 c_{1} c_{3}\left(5 \alpha^{2} c_{2}^{3} c_{4}+c_{2}^{2}\left(6+\alpha^{2}\left(-5 g+18 c_{3}\right) c_{4}\right)\right. \\
+3 c_{3}\left(4 c_{3}+\left(-60+\alpha^{2} c_{3}^{2}\right) c_{4}\right) \\
+c_{2}\left(-6\left(-g+60 c_{4}\right)\right. \\
\left.\left.+c_{3}\left(12+\alpha^{2}\left(-5 g+16 c_{3}\right) c_{4}\right)\right)\right) \\
+4 c_{1}^{2}\left(c_{2}^{2}\left(1+25 \alpha^{2} c_{4}^{2}\right)\right. \\
+2\left(c_{3}^{2}+60 c_{3} c_{4}+30\left(-30+\alpha^{2} c_{3}^{2}\right) c_{4}^{2}\right) \\
+c_{2}\left(-g\left(-1+25 \alpha^{2} c_{4}^{2}\right)\right. \\
\left.\left.\left.+2 c_{3}\left(1+55 \alpha^{2} c_{4}^{2}\right)\right)\right)\right) .
\end{gathered}
$$

In this case, the generalized soliton solution can be written as

$$
\begin{aligned}
\eta_{3}(x, t)= & \frac{-1}{4 \alpha c_{1}\left(c_{3}\left(c_{2}+c_{3}\right)-10 c_{1} c_{4}\right)} \\
& \cdot\left(c_{3}\left(6+\alpha\left(c_{2}+c_{3}\right)\right)\left(c_{2}+2 c_{3}-g\right)\right. \\
& \quad-2 c_{1}\left(-g+5\left(12-g \alpha+2 \alpha c_{3}\right) c_{4}\right. \\
& \left.\left.\quad+c_{2}\left(-1+5 \alpha c_{4}\right)\right)\right) \\
+ & \frac{3\left(c_{2}+2 c_{3}-g\right)}{2 c_{1}} \operatorname{sech}^{2}[x-k t] \\
\pm & \frac{3}{\sqrt{2} c_{1}} \cdot \sqrt{-\left(c_{2}+2 c_{3}\right)^{2}+20 c_{1} c_{4}+\left(c_{2}+c_{3}\right) g} \\
& \times \operatorname{sech}[x-k t] \tanh [x-k t] .
\end{aligned}
$$

3.2.2. Using the Extended tanh Method. We have

$$
u(\xi)=a_{0}+a_{1} Y+a_{2} Y^{2}+\frac{b_{1}}{Y}+\frac{b_{2}}{Y^{2}} .
$$

By substituting (33) into (23) and collecting the coefficient of $Y$, we obtain a system of algebraic equations for $a_{0}, a_{1}, a_{2}, b_{1}$, $b_{2}$, and $k$. Solving this system gives the following solution.

Case I. Consider

$$
\begin{aligned}
a_{0}= & -6 \pm 4 g \mu^{2} \alpha+4 \mu^{2} \alpha\left(c_{2}+2 c_{3}\right) \\
& \left.+\frac{\left( \pm g-c_{2}\right)\left(c_{1}-3 c_{3}\right)}{c_{3}\left(c_{2}+c_{3}\right)-10 c_{1} c_{4}}\right) \times\left(2 \alpha c_{1}\right)^{-1} \\
a_{1}=b_{1}= & b_{2}=0, \quad a_{2}=-\frac{3 \mu^{2}\left( \pm g+c_{2}+2 c_{3}\right)}{c_{1}} \\
k= & \frac{-9+2 \mu^{4} \alpha^{2} c_{2}\left( \pm g+c_{2}+2 c_{3}\right)}{c_{1} \alpha} \\
& -24 \mu^{4} \alpha c_{4}-\frac{\left( \pm g-c_{2}\right) c_{2}\left(c_{1}-3 c_{3}\right)^{2}}{2 \alpha c_{1}\left(c_{3}\left(c_{2}+c_{3}\right)-10 c_{1} c_{4}\right)^{2}} \\
& +\frac{\left(c_{1}-3 c_{3}\right)^{2}}{\alpha c_{1}\left(c_{3}\left(c_{2}+c_{3}\right)-10 c_{1} c_{4}\right)} .
\end{aligned}
$$

In this case, the generalized soliton solution can be written as

$$
\begin{aligned}
\eta_{1}(x, t)= & \left(-6 \pm 4 g \mu^{2} \alpha+4 \mu^{2} \alpha\left(c_{2}+2 c_{3}\right)\right. \\
& \left.+\frac{\left( \pm g-c_{2}\right)\left(c_{1}-3 c_{3}\right)}{c_{3}\left(c_{2}+c_{3}\right)-10 c_{1} c_{4}}\right) \times\left(2 \alpha c_{1}\right)^{-1} \\
& -\frac{3 \mu^{2}\left( \pm g+c_{2}+2 c_{3}\right)}{c_{1}} \tanh ^{2}[\mu(x-k t)],
\end{aligned}
$$

where $g=\sqrt{\left(c_{2}+2 c_{3}\right)^{2}-40 c_{1} c_{4}}$ and $\left(c_{2}+2 c_{3}\right)^{2}>40 c_{1} c_{4}$.

Figure 4(a) shows the stability bright solitary wave solutions with $(\alpha=-1)$ in the interval $[-5,5]$ and time in the interval $[0,0.5]$. 


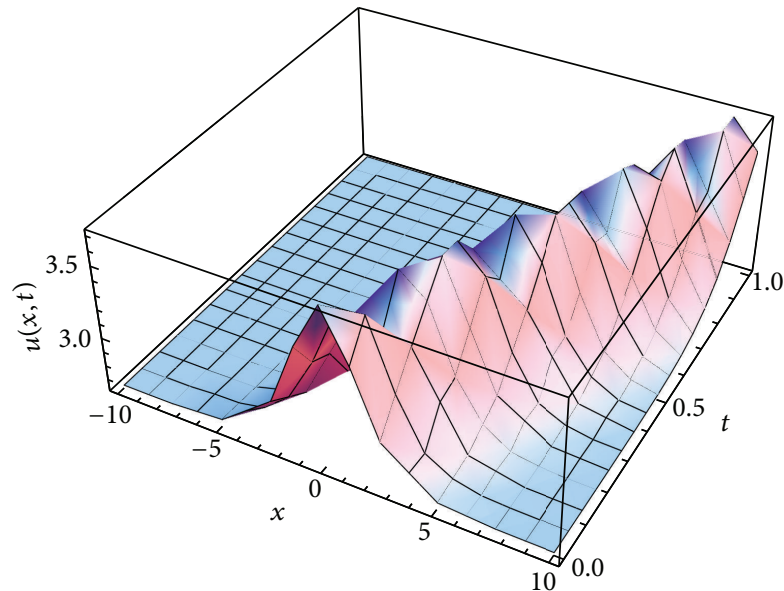

(a)

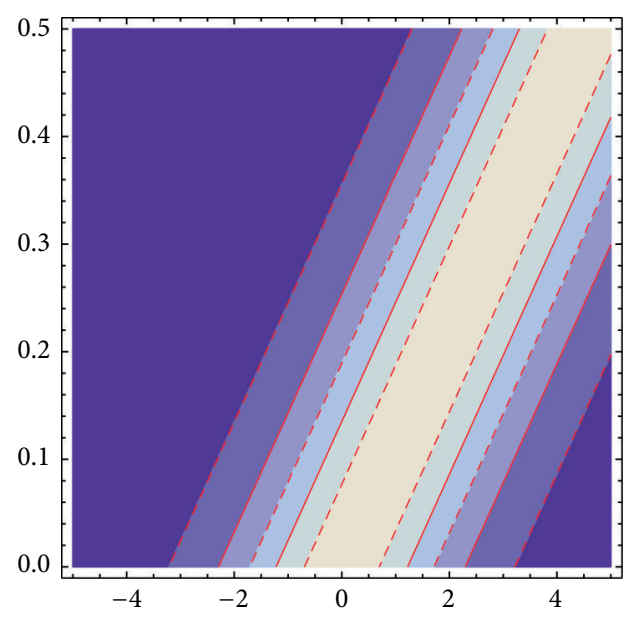

(b)

FIGURE 4: (a) Travelling waves solutions of (35) is plotted: stability bright solitary waves. (b) Travelling waves solutions of (35) is plotted: stability contour of solitary waves.

Figure 4(b) shows the stability contour of solitary wave solution with $(\alpha=-1)$ in the interval $[-5,5]$ and time in the interval $[0,0.5]$.

\section{Case II. Consider}

$$
\begin{aligned}
a_{0}= & -6 \pm 4 g \mu^{2} \alpha+4 \mu^{2} \alpha\left(c_{2}+2 c_{3}\right) \\
& \left.+\frac{\left( \pm g-c_{2}\right)\left(c_{1}-3 c_{3}\right)}{c_{3}\left(c_{2}+c_{3}\right)-10 c_{1} c_{4}}\right) \times\left(2 \alpha c_{1}\right)^{-1}, \\
a_{1}= & b_{1}=0, \quad a_{2}=b_{2}=-\frac{3 \mu^{2}\left( \pm g+c_{2}+2 c_{3}\right)}{c_{1}}, \\
k= & \frac{-9+32 \mu^{4} \alpha^{2} c_{2}\left( \pm g+c_{2}+2 c_{3}\right)}{c_{1} \alpha}-384 \mu^{4} \alpha c_{4} \\
& -\frac{\left( \pm g-c_{2}\right) c_{2}\left(c_{1}-3 c_{3}\right)^{2}}{2 \alpha c_{1}\left(c_{3}\left(c_{2}+c_{3}\right)-10 c_{1} c_{4}\right)^{2}} \\
& +\frac{\left(c_{1}-3 c_{3}\right)^{2}}{\alpha c_{1}\left(c_{3}\left(c_{2}+c_{3}\right)-10 c_{1} c_{4}\right)} .
\end{aligned}
$$

In this case, the generalized soliton solution can be written as

$$
\begin{aligned}
\eta_{2}(x, t)=( & -6 \pm 4 g \mu^{2} \alpha+4 \mu^{2} \alpha\left(c_{2}+2 c_{3}\right) \\
& \left.+\frac{\left( \pm g-c_{2}\right)\left(c_{1}-3 c_{3}\right)}{c_{3}\left(c_{2}+c_{3}\right)-10 c_{1} c_{4}}\right) \times\left(2 \alpha c_{1}\right)^{-1} \\
& -\frac{3 \mu^{2}\left( \pm g+c_{2}+2 c_{3}\right)}{c_{1}} \\
& \times\left(\tanh ^{2}[\mu(x-k t)]+\operatorname{coth}^{2}[\mu(x-k t)]\right) .
\end{aligned}
$$

Figure 5(a) shows the stability bright solitary wave solutions with $(\alpha=-1)$ in the interval $[-5,5]$ and time in the interval $[0,0.5]$.
Figure 5(b) shows the stability contour of solitary wave solution with $(\alpha=-1)$ in the interval $[-5,5]$ and time in the interval $[0,0.5]$.

Case III. Consider

$$
\begin{gathered}
a_{0}=\left(-6 \pm 4 g \mu^{2} \alpha+4 \mu^{2} \alpha\left(c_{2}+2 c_{3}\right)\right. \\
\left.+\frac{\left( \pm g-c_{2}\right)\left(c_{1}-3 c_{3}\right)}{c_{3}\left(c_{2}+c_{3}\right)-10 c_{1} c_{4}}\right) \times\left(2 \alpha c_{1}\right)^{-1} \\
a_{1}=a_{2}=b_{1}=0, \quad b_{2}=-\frac{3 \mu^{2}\left( \pm g+c_{2}+2 c_{3}\right)}{c_{1}}, \\
k=\frac{-9+2 \mu^{4} \alpha^{2} c_{2}\left( \pm g+c_{2}+2 c_{3}\right)}{c_{1} \alpha}-24 \mu^{4} \alpha c_{4} \\
-\frac{\left( \pm g-c_{2}\right) c_{2}\left(c_{1}-3 c_{3}\right)^{2}}{2 \alpha c_{1}\left(c_{3}\left(c_{2}+c_{3}\right)-10 c_{1} c_{4}\right)^{2}} \\
+\frac{\left(c_{1}-3 c_{3}\right)^{2}}{\alpha c_{1}\left(c_{3}\left(c_{2}+c_{3}\right)-10 c_{1} c_{4}\right)} .
\end{gathered}
$$

In this case, the generalized soliton solution can be written as

$$
\begin{aligned}
\eta_{3}(x, t)=( & -6 \pm 4 g \mu^{2} \alpha+4 \mu^{2} \alpha\left(c_{2}+2 c_{3}\right) \\
& \left.+\frac{\left( \pm g-c_{2}\right)\left(c_{1}-3 c_{3}\right)}{c_{3}\left(c_{2}+c_{3}\right)-10 c_{1} c_{4}}\right) \times\left(2 \alpha c_{1}\right)^{-1} \\
& -\frac{3 \mu^{2}\left( \pm g+c_{2}+2 c_{3}\right)}{c_{1}} \operatorname{coth}^{2}[\mu(x-k t)] .
\end{aligned}
$$

Figure 6(a) shows the stability bright solitary wave solutions with $(\alpha=-1)$ in the interval $[-5,5]$ and time in the interval $[0,0.5]$. Figure $6(\mathrm{~b})$ shows the stability contour of solitary wave solution with $(\alpha=-1)$ in the interval $[-5,5]$ and time in the interval $[0,0.5]$. 


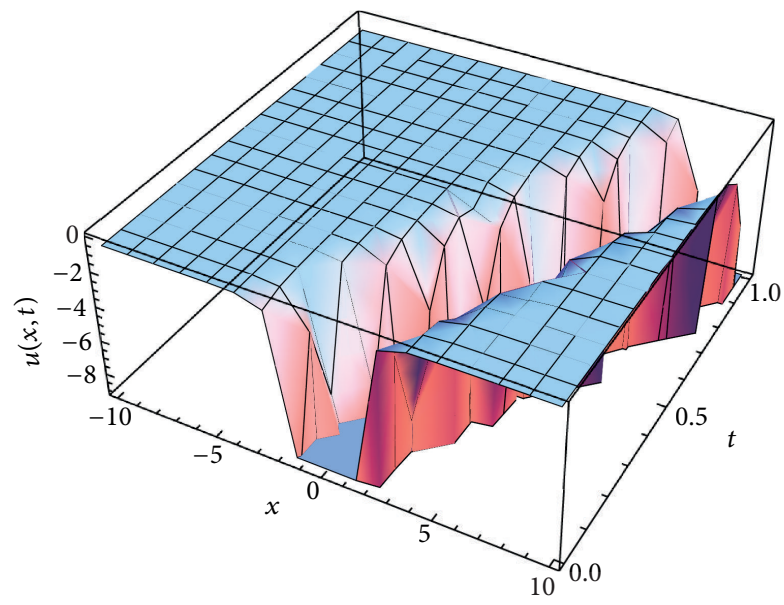

(a)

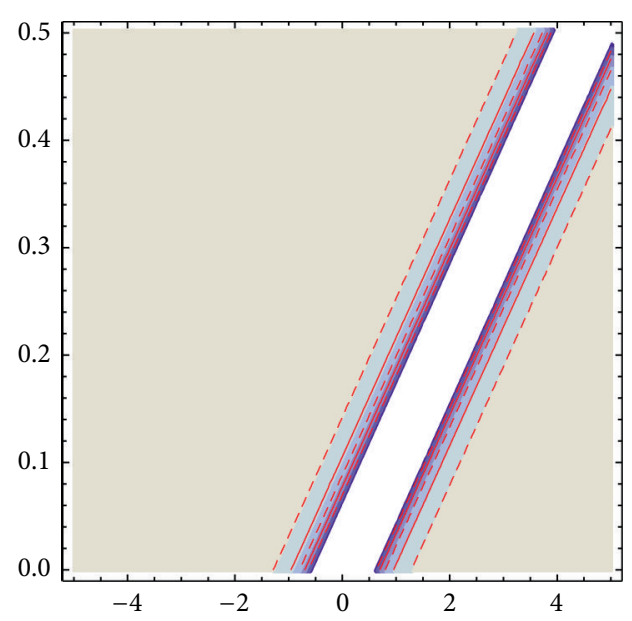

(b)

FIGURE 5: (a) Travelling waves solutions of (37) is plotted: stability dark solitary waves. (b) Travelling waves solutions of (37) is plotted: stability contour of solitary waves.

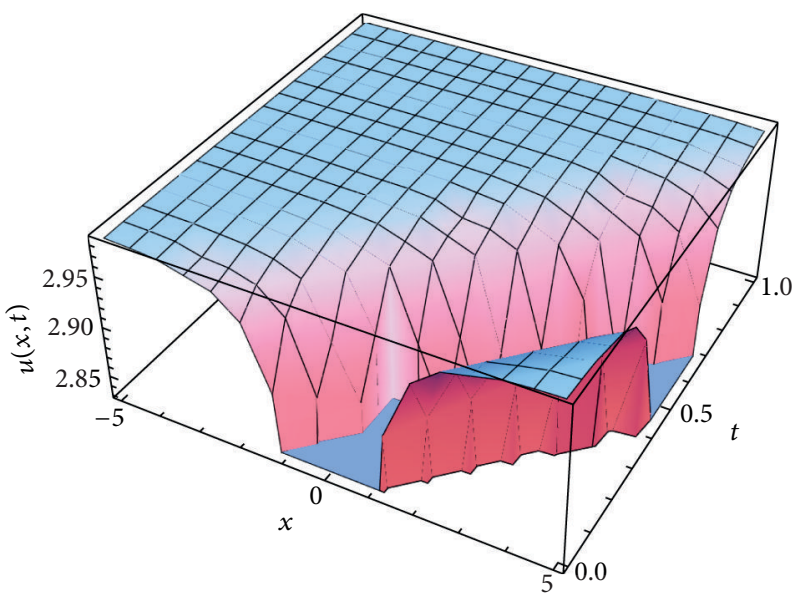

(a)

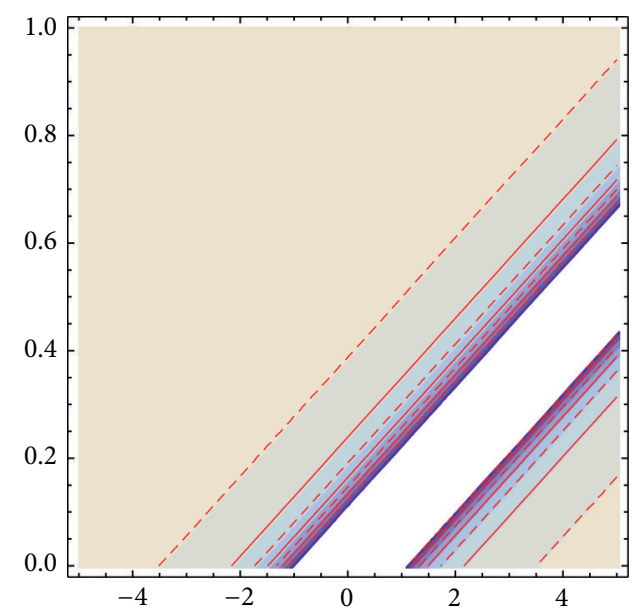

(b)

Figure 6: (a) Travelling waves solutions of (39) is plotted: stability dark solitary waves. (b) Travelling waves solutions of (39) is plotted: stability contour of solitary waves.

\section{Conclusion}

Contour plots produced by Mathematica are drawn shaded, in such a way that regions with higher values of $u_{i}(x, t)$, $v_{i}(x, t)$, and $\eta_{i}(x, t), i=1,2,3$, are drawn lighter. As with all Mathematica graphics commands, options allow you to control the appearance of the graph. Contours plot allows you to determine the number of contours to be drawn. The default is ten equally spaced curves. An analytic study was conducted on the Olver and fifth-order $\mathrm{KdV}$ equations. We formally derived travelling wave solutions for the Olver and fifth-order $\mathrm{KdV}$ equations. However, by using another distinct approach, we derived one traveling wave solutions for each Olver and fifth-order $\mathrm{KdV}$ equations. The structures of the obtained solutions are distinct and stable.

\section{Conflict of Interests}

The authors declare that there is no conflict of interests regarding the publication of this paper.

\section{Acknowledgment}

This project was supported by the Deanship of Scientific Research, Taibah University, KSA, Project no. 4086/1434, year 2013. 


\section{References}

[1] L. Jin, "Application of variational iteration method to the fifthorder KdV equation," International Journal of Contemporary Mathematical Sciences, vol. 3, no. 5, pp. 213-221, 2008.

[2] A. H. Khater, M. M. Hassan, and R. S. Temsah, "Cnoidal wave solutions for a class of fifth-order KdV equations," Mathematics and Computers in Simulation, vol. 70, no. 4, pp. 221-226, 2005.

[3] T. Kawahara, "Oscillatory solitary waves in dispersive media," Journal of the Physical Society of Japan, vol. 33, pp. 260-264, 1972.

[4] M. A. Helal, "Soliton solution of some nonlinear partial differential equations and its applications in fluid mechanics," Chaos, Solitons and Fractals, vol. 13, no. 9, pp. 1917-1929, 2002.

[5] R. R. Long, "Solitary waves in one- and two fluids systems," Tellus, vol. 8, Article ID 460471, 1956.

[6] D. J. Benney, "Long nonlinear waves in fluid flows," Journal of Mathematical Physics, vol. 45, article 5263, 1966.

[7] R. S. Drazin and P. G. Johnson, Solutions: An Introduction, Cambridge University Press, Cambridge, UK, 1989.

[8] V. I. Karpman, "Lyapunov approach to the soliton stability in highly dispersive systems. II. KdV-type equations," Physics Letters A, vol. 215, no. 5-6, pp. 257-259, 1996.

[9] X. Liu and C. Bai, "Exact solutions of some fifth-order nonlinear equations," Applied Mathematics B, vol. 15, no. 1, pp. 28-32, 2000.

[10] E. J. Parkes and B. R. Duffy, "An automated Tanh-function method for finding solitary wave solutions to non-linear evolution equations," Computer Physics Communications, vol. 98, pp. 288-300, 1996

[11] A. M. Wazwaz, "Compactons and solitary patterns solutions to fifth-order KdV like equations," Physica A, vol. 371, pp. 273-279, 2006.

[12] A.-M. Wazwaz, "Abundant solitons solutions for several forms of the fifth-order KdV equation by using the tanh method," Applied Mathematics and Computation, vol. 182, no. 1, pp. 283300, 2006.

[13] A. Biswas and S. Konar, "Soliton perturbation theory for the compound KdV equation," International Journal of Theoretical Physics, vol. 46, no. 2, pp. 237-243, 2007.

[14] A. Biswas and E. Zerrad, "Soliton perturbation theory for the generalized fifth-order nonlinear equation," Contemporary Engineering Science, vol. 1, no. 2, pp. 63-69, 2008.

[15] M. A. Helal and A. R. Seadawy, "Variational method for the derivative nonlinear Schrödinger equation with computational applications," Physica Scripta, vol. 80, pp. 350-360, 2009.

[16] M. A. Helal and A. R. Seadawy, "Exact soliton solutions of a D-dimensional nonlinear Schrödinger equation with damping and diffusive terms," Zeitschrift für Angewandte Mathematik und Physik, vol. 62, no. 5, pp. 839-847, 2011.

[17] A. R. Seadawy, "New exact solutions for the KdV equation with higher order nonlinearity by using the variational method," Computers \& Mathematics with Applications, vol. 62, no. 10, pp. 3741-3755, 2011.

[18] A. R. Seadawy, "Exact solutions of a two-dimensional nonlinear Schrödinger equation," Applied Mathematics Letters, vol. 25, no. 4, pp. 687-691, 2012.

[19] A. R. Seadawy, "Traveling wave solutions of the Boussinesq and generalized fifth-order KdV equations by using the direct algebraic method," Applied Mathematical Sciences, vol. 6, pp. 4081-4090, 2012.
[20] M. A. Helal and A. R. Seadawy, "Benjamin-Feir instability in nonlinear dispersive waves," Computers \& Mathematics with Applications, vol. 64, no. 11, pp. 3557-3568, 2012.

[21] D. Kaya, "An explicit and numerical solutions of some fifthorder KdV equation by decomposition method," Applied Mathematics and Computation, vol. 144, no. 2-3, pp. 353-363, 2003.

[22] P. J. Olver, "Hamiltonian and non-Hamiltonian models for water waves," in Trends and Applications of Pure Mathematics to Mechanics, vol. 195 of Lecture Notes in Physics, pp. 273-290, Springer, Berlin, Germany, 1984.

[23] N. A. Kudryashov, M. B. Soukharev, and M. V. Demina, "Elliptic traveling waves of the Olver equation," Communications in Nonlinear Science and Numerical Simulation, vol. 17, no. 11, pp. 4104-4114, 2012.

[24] A. R. Seadawy and K. El-Rashidy, "Traveling wave solutions for some coupled nonlinear evolution equations," Mathematical and Computer Modelling, vol. 57, no. 5-6, pp. 1371-1379, 2013.

[25] M. A. Helal, A. R. Seadawy, and R. S. Ibrahim, "Variational principle for Zakharov-Shabat equations in two-dimensions," Applied Mathematics and Computation, vol. 219, no. 10, pp. 5635-5648, 2013.

[26] A. R. Seadawy and A. Sayed, "Travelling wave solution of two-dimensional nonlinear KdV-Burgers equation," Applied Mathematical Sciences, vol. 7, no. 68, pp. 3367-3377, 2013.

[27] M. A. Helal, A. R. Seadawy, and M. H. Zekry, "Stability analysis solutions for the fourth-order nonlinear AblowitzKaup-Newell-Segur water wave equation," Applied Mathematical Sciences, vol. 7, no. 65-68, pp. 3355-3365, 2013.

[28] A. R. Seadawy, "Stability analysis for Zakharov-Kuznetsov equation of weakly nonlinear ion-acoustic waves in a plasma," Computers \& Mathematics with Applications, vol. 67, no. 1, pp. 172-180, 2014. 


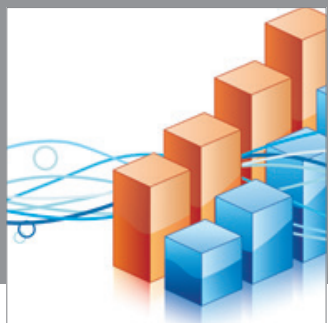

Advances in

Operations Research

mansans

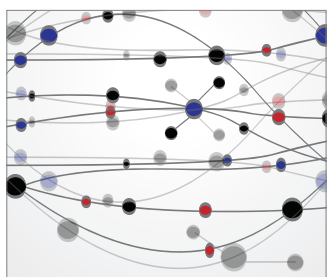

The Scientific World Journal
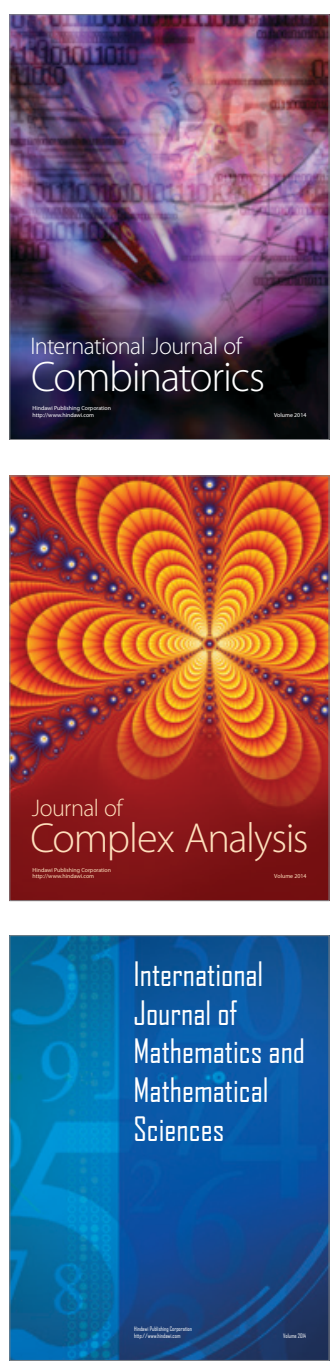
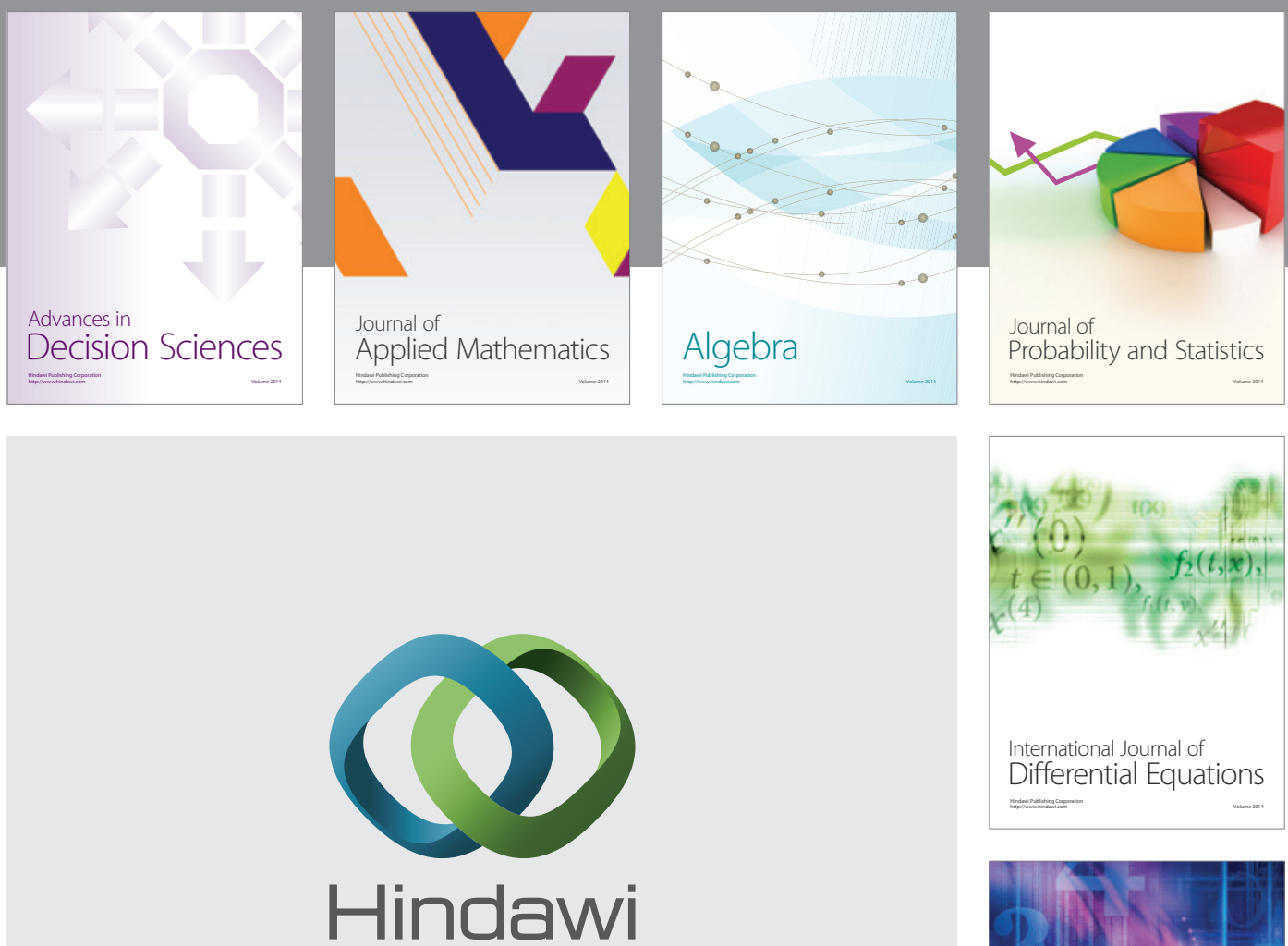

Submit your manuscripts at http://www.hindawi.com
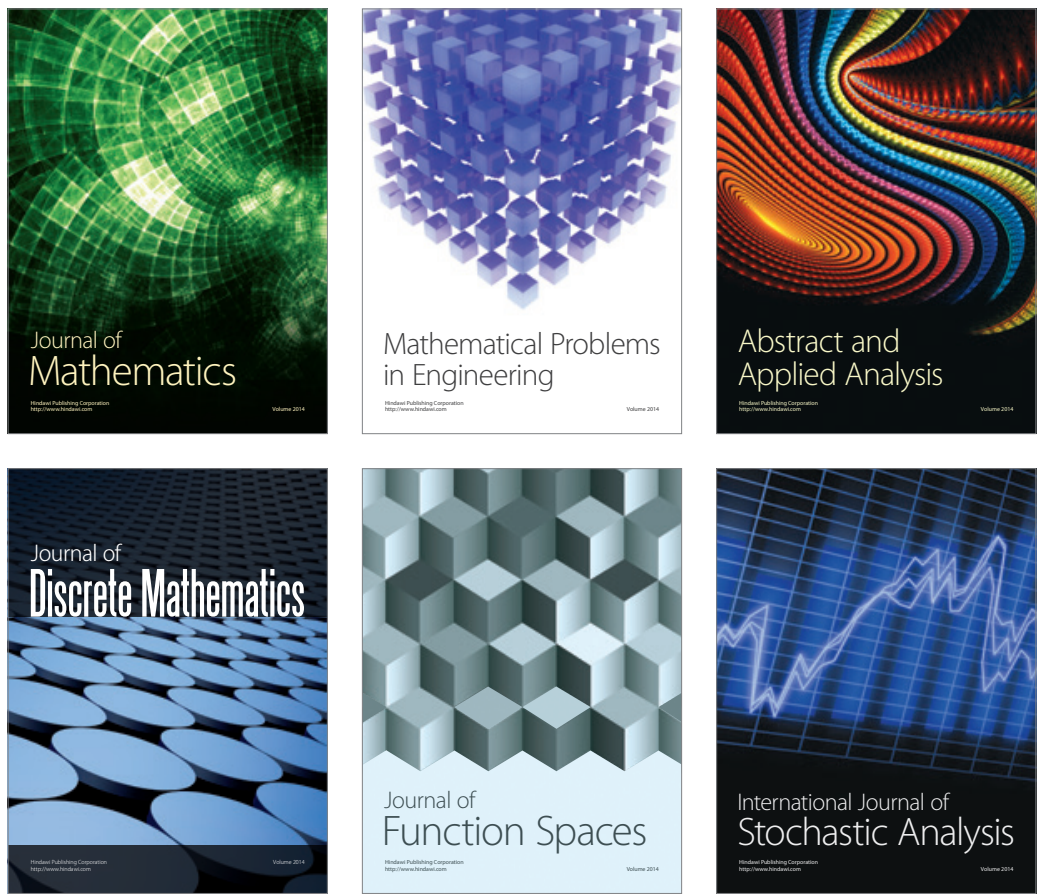

Journal of

Function Spaces

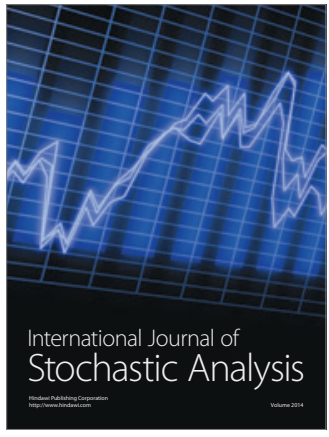

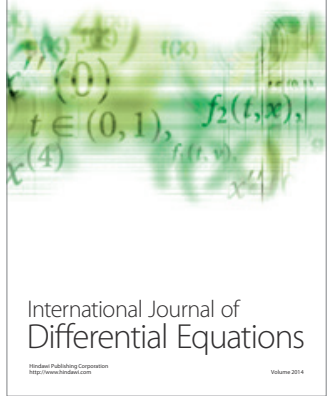
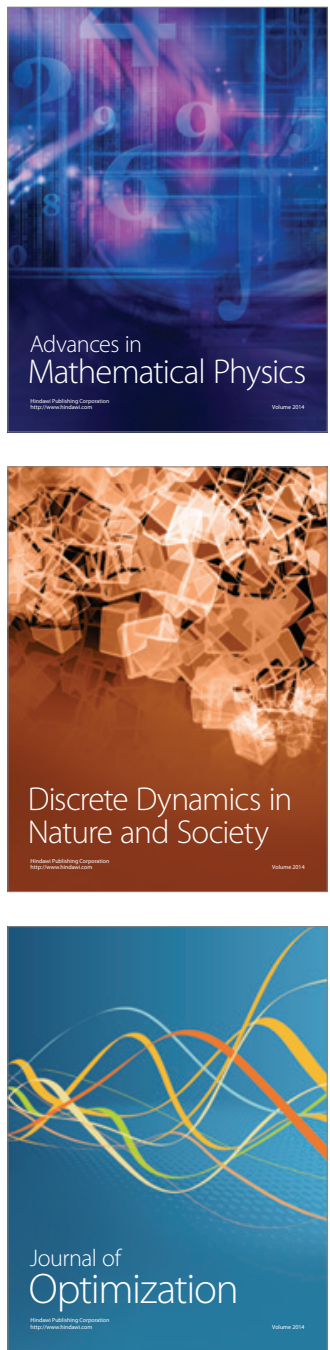